\title{
Computed Tomography in the Evaluation of Plexopathies and Proximal Neuropathies
}

\author{
John D. Stewart, Brian Schmidt and Roberto Wee
}

SUMMARY: We describe nine patients with plexopathies or proximal mononeuropathies due to mass lesions. In four, computed tomography (CT) was the only radiological technique to show the cause of the neuropathy. In five patients, CT either unequivocally confirmed the presence of an abnormality or was superior to other imaging techniques in showing its full anatomical extent. CT scanning is a valuable aid in the assessment of lesions of the peripheral nervous system, particularly plexopathies and mononeuropathies caused by retroperitoneal, pelvic or superior pulmonary sulcus tumors.

RÉSUMÉ: Nous présentons neuf (9) patients porteurs de plexopathies ou de mononeuropathies secondaires à des lésions occupant de l'espace. Chez quatre (4) d'entre eux, la scannographie (CT) s'est avérée la seule modalité radiologique capable de démontrer la cause de la neuropathie. Chez cinq (5) autres patients, la scannographie (CT) a confirmé de façon non équivoque la présence d'une anomalie, ou s'est montrée supérieure à toute autre modalité d'investigation pour en définir toute l'extension anatomique. La scannographie (CT) s'est avérée très utile pour évaluer les lésions du système nerveux périphérique, en particulier les plexopathies et les mononeuropathies causées par des tumeurs du rétropéritoine, du bassin et du sulcus pulmonaire supérieur.

Can. J. Neurol. Sci. 1983; 10:244-247

Computed tomography (CT) is firmly established as an important diagnostic tool for the investigation of lesions within the cranium, thorax and abdomen. However, less attention has been given to its role in the evaluation of the peripheral nervous system. The present report describes our experience with CT scanning in nine patients with a variety of mass lesions affecting the plexuses or proximal nerves. In four patients CT was the only diagnostic procedure to demonstrate the abnormality, while in five patients the full anatomical extent or unequivocal presence of an abnormality was appreciated only after CT scanning.

\section{CASE RePORTS}

Lumbar and sacral nerve root or plexus syndromes

Patient 1:

A 22 year old man had intermittent low back pain of increasing severity for one year and then had difficulty initiating micturition. Examination showed marked impairment of right straight leg raising. Right plantar flexion, inversion and toe clawing were mildly weak. The right ankle reflex was absent. Light touch and pinprick sensation were diminished over the lateral border of the right foot and the posterior surface of the leg to the gluteal fold. Anal sphincter tone was normal.

The clinical diagnoses included sacral radiculopathy, lumbo-sacral plexopathy or sciatic neuropathy. Electromyographic (EMG) studies showed amongst other abnormalities, denervation of the lumbo-sacral paraspinal muscles, indicating nerve root involvement. Plain radiographs of the pelvis showed a possible abnormality of the right sacrum obscuring the SI-S3 nerve root foramena, and a technetium bone scan showed abnormal uptake over the right sacrum. An intravenous pyelogram and a myelogram were normal. A CT scan showed a large destructive lesion involving the right sacral wing with a soft tissue mass projecting anteriorly (Fig. 1). At laparotomy a Ewing's sarcoma was found to be protruding from the right sacrum.

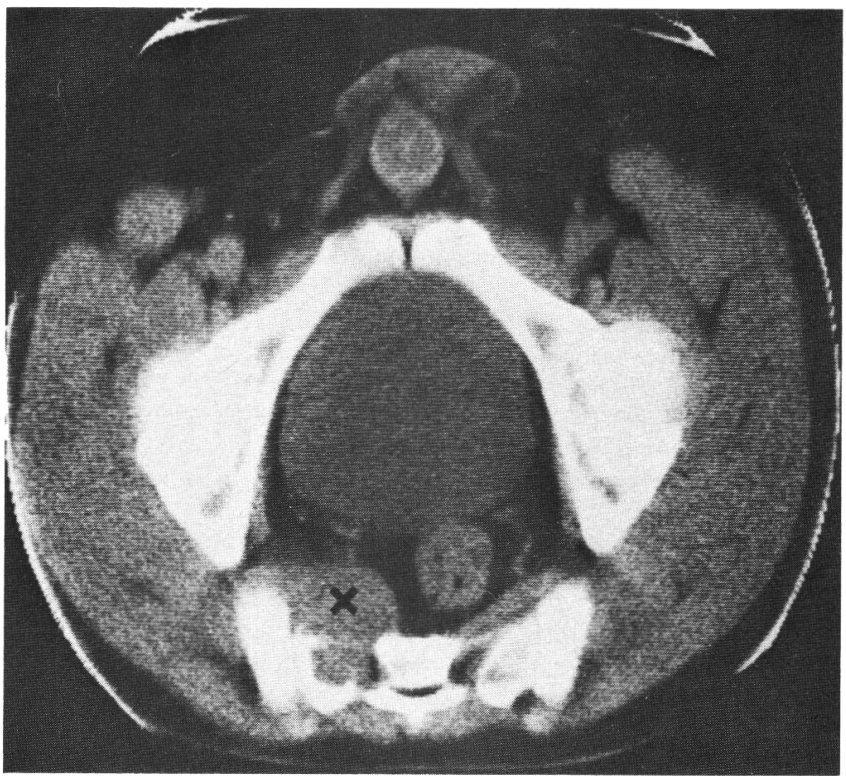

Figure I-Patient I.CT scan of pelvis: A soft tissue mass (Ewing's sarcoma) involving sacrum is indicated by ' $x$ '.

Patient 2:

A 70 year old male had a two month history of low back pain and perianal numbness. There was no disturbance of micturition. Neurological examination was normal except for decreased sensation in the S3-5 dermatomes bilaterally. Rectal examination was normal.

Plain radiographs of the lumbosacral spine and pelvis showed marked osteolytic destruction of the sacrum. A myelogram, intravenous pyelogram and barium enema were normal. CT scanning revealed a destructive

From the Departments of Neurology and Neurosurgery, and Radiology, McGill University and The Montreal General Hospital, Montreal.

Received May 31, 1983. Accepted July 21, 1983.

Reprint requests to: Dr. John D. Stewart, Division of Neurology, The Montreal General Hospital, I650 Cedar Avenue, Montreal, Quebec H3G IA4, Canada. 
lesion mainly involving the left side of the sacrum with a soft tissue mass extending anteriorly (Fig. 2). A subsequent technetium bone scan showed increased uptake in several areas including the ribs, thoracic spine and sacrum. The prostatic acid phosphatase was markedly elevated and he was presumed to have metastatic carcinoma of the prostate.

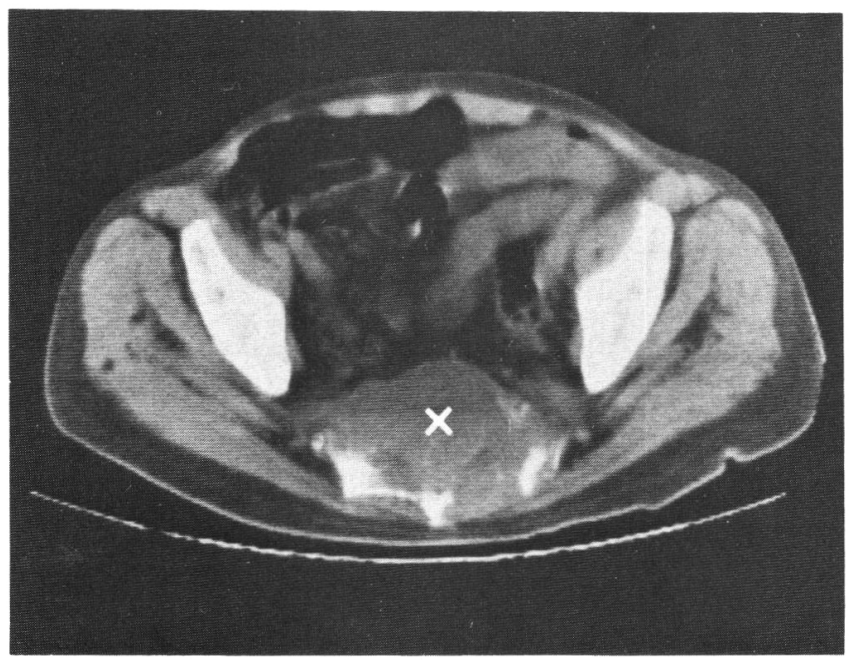

Figure 2 - Patient 2. CT scan of pelvis: $A$ large mass (presumed metastatic carcinoma of the prostate) destroying the sacrum is indicated by ' $x$ '.

\section{Patient 3:}

A 57 year old man had a four month history of pain in the right buttock and posterior thigh, with burning dysesthesias over the lateral aspect of the right lower leg and foot. Examination showed weakness of right plantar flexion, an absent right ankle jerk, and diminished sensation in L5, SI dermatomes. The anal tone and prostate gland were clinically normal.

On plain lumbo-sacral radiographs the margins of the right S1 and S2 anterior nerve root foraminae were indistinct. A myelogram showed mild antero-lateral compression of the dural sac at the level of S2. A technetium bone scan demonstrated increased uptake in the right sacrum, scapula, skull and ribs. The $\mathrm{CT}$ scan revealed a mass lying adjacent to the right side of the sacrum with extension into the sacral epidural space. A biopsy showed this to be adenocarcinoma of the prostate.

\section{Patient 4a:}

A 57 year old woman presented with a 5 month history of low back pain radiating into the left groin and thigh. Five and two years previously, she had undergone mastectomies for carcinomas of the breasts. One year prior to this admission she underwent a hysterectomy and pelvic radiotherapy for stage III endometrial carcinoma.

On examination mild weakness of the left iliopsoas, quadriceps and tibialis anterior was found. The left knee reflex was diminished as was sensation over the anterior thigh. EMG evaluation showed changes of denervation in L2-L5 innervated muscles, sparing the paraspinal muscles. A diagnosis of an infiltrating malignant lesion of the lumbar plexus was made.

Lumbo-sacral spine radiographs and an isotope bone scan were normal. An intravenous pyelogram showed no displacement of the ureters. A CT scan showed bulky retroperitoneal soft tissue masses. predominantly on the left side, presumably metastatic endometrial carcinoma of the para-aortic lymph nodes. Radiotherapy produced a remission of her neurological signs and symptoms.

\section{Lower trunk brachial plexus syndromes}

\section{Patient 4b:}

The patient described as 4a later developed pain in the left axilla and medial aspect of the arm, associated with numbness of the medial forearm and 4th and 5th digits. Examination showed mild weakness of the small muscles of the left hand and diminished sensation in the above distribution. A diagnosis of metastatic infiltration of the lower trunk of the brachial plexus was made. Chest and cervical spine radiographs were normal, as was the isotope bone scan. However, a CT scan showed a soft tissue mass deep to the left scalenus anterior muscle. A supraclavicular node biopsy showed adenocarcinoma consistent with a primary neoplasm of the breast. Radiotherapy to this area produced improvement in her signs and symptoms.

\section{Patient 5:}

A 53 year old male heavy smoker had pain in his right upper chest and shoulder for 10 months, associated with numbness of the right fourth and fifth digits and medial forearm. Examination showed a right Horner's syndrome, wasting of the intrinsic muscles of the right hand, with diminished sensation in the fourth and fifth digits and ulnar border of the forearm. Electromyography (EMG) showed denervation in muscles supplied by the right $\mathrm{C} 8$ and $\mathrm{TI}$ nerve roots and lower trunk of the brachial plexus.

Chest radiographs with apical views, cervical and thoracic spine radiographs were all normal, as was a technetium bone scan. A CT scan demonstrated a soft tissue mass in the apex of the right lung (Fig. 3). At surgical exploration a poorly differentiated adenocarcinoma was found to be adherent to the lower trunk of the brachial plexus.

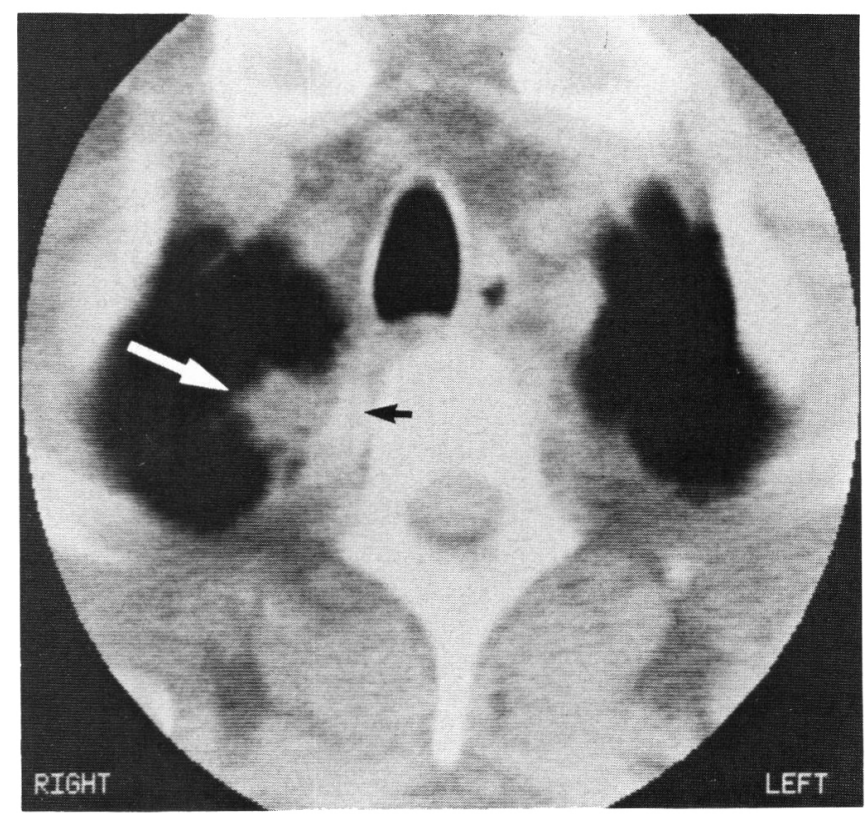

Figure 3 - Patient 5. CT scan at level of first thoracic versebra: White arrow indicates soft tissue mass (adenocarcinoma) in apex of right lung adjacent to the head of the first rib (black arrow).

\section{Patient 6:}

A 49 year old heavy smoker presented with numbness and pain in the medial aspect of his left arm and forearm. Neurological examination showed a left Horner's syndrome, and decreased sensation over the medial aspect of the left forearm.

A chest radiograph with special views of the apices, cervical spine radiographs and a technetium bone scan were all normal. A CT scan demonstrated a soft tissue mass in the apex of the left lung, similar to that in patient 5 . Radiotherapy rapidly relieved the patient's symptoms. Hepatomegaly developed later and a liver biopsy showed poorly differentiated adenocarcinoma, presumably metastatic from lung.

\section{Proximal peripheral nerve syndromes}

Patient 7:

A 33 year old woman presented with two years of pain in the low back, right buttock and posterior thigh, often most severe the day before menstruation. One year later a myelogram was normal. During the subsequent months the leg became progressively weaker.

Examination revealed diffuse wasting of the right lower limb, most marked in the gluteus maximus. The right gluteal fold area was exquisitely tender on deep palpation. Power was moderately reduced in the right gluteus maximus, hamstrings, foot dorsiflexors and evertors. Light touch sensation was reduced over the dorsolateral aspect of the right 
foot. Nerve conduction and EMG studies supported the clinical diagnosis of lesions of the sciatic and inferior gluteal nerves in the region of the sciatic notch. Radiographs of the lumbo-sacral spine, pelvis and right hip, and another myelogram were all normal. A CT scan showed a soft tissue mass in the right sciatic notch (Fig. 4).

At surgical exploration, a hemorrhagic mass encasing a swollen sciatic nerve was found in the sciatic notch. The pyriformis and gemelli muscles were scarred and were also compressing the nerve. These muscles were divided and the mass was partially removed: pathological evaluation showed it to be endometriosis.

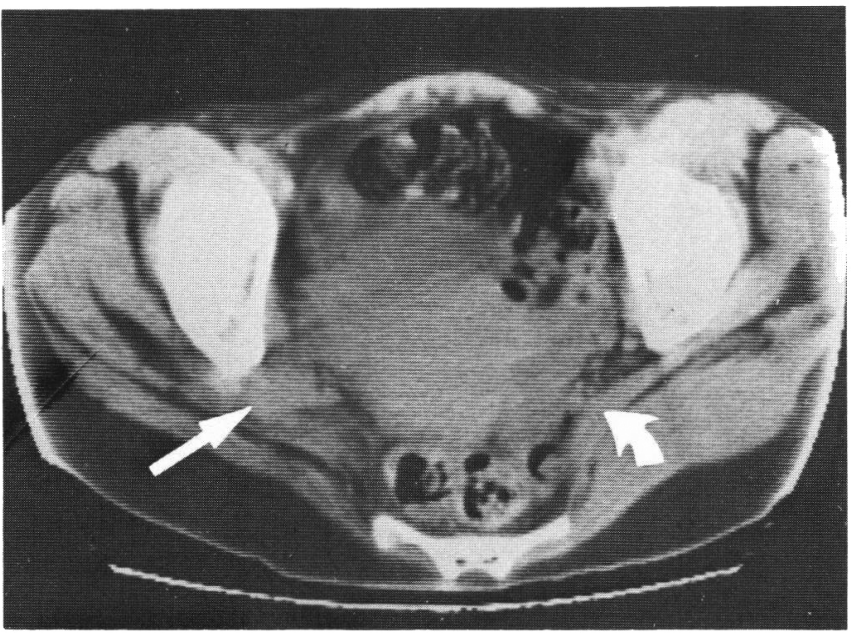

Figure 4-Patient 7.CT scan of pelvis at level of sciatic notch: Curved arrow indicates normal pyriformis muscle on uninvolved side. Straight arrow indicates endometriosis in the sciatic notch. (Stewart et. al., 1981).

\section{Patient 8:}

A 16 year old male developed groin pain, paresthesia over the anterior surface of the left thigh and antero-lateral aspect of the lower leg, and gradual wasting of the left thigh.

Examination showed mild weakness and areflexia of the left quadriceps, with pinprick and light touch sensation diminished in the area described above. A diagnosis of an L3 radiculopathy was made. Lumbo-sacral spine and hip radiographs showed only a faint bony bar of uncertain significance arising from the left iliac wing. A myelogram was normal. The $\mathrm{CT}$ scan demonstrated a pedunculated bony growth arising from the left iliac bone protruding into the iliacus muscle (Fig. 5). At operation

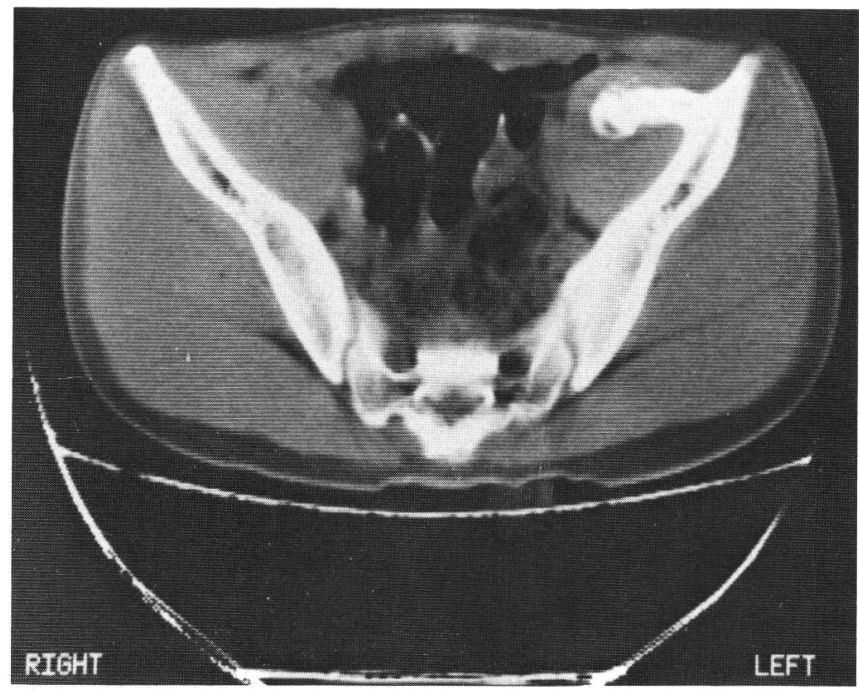

Figure 5 - Patient 8. CT scan of pelvis at level of anterior superior iliac spine: A prominent bony growth with an ossified cartilagenous cap (osteochondroma) is seen arising from the left iliac bone. the femoral and lateral femoral cutaneous nerves were found to be hooked over this bony bar which was then excised. Histological examination confirmed the radiological diagnosis of an osteochondroma.

\section{Patient 9:}

A 20 year old male had numbness over the posterior surface of the left thigh for one year. On examination there was mildly diminished sensation in the distribution of the posterior cutaneous nerve of the thigh. Radiographs of the lumbo-sacral spine and the pelvis were normal except for questionable indistinctness of the margin of the left second sacral intervertebral foramen. A myelogram and an intravenous pyelogram were normal. A technetium bone scan showed some increased activity of uncertain significance in the left femoral head. CT scanning revealed a mass in the left presacral region (Fig. 6). On surgical exploration a highly vascular tumor was found under the pyriformis muscle invading the left side of the sacrum and presumably compressing the posterior cutaneous nerve of the thigh, although this was not specifically identified at the time of the operation. The histological diagnosis was hemangiopericytoma.

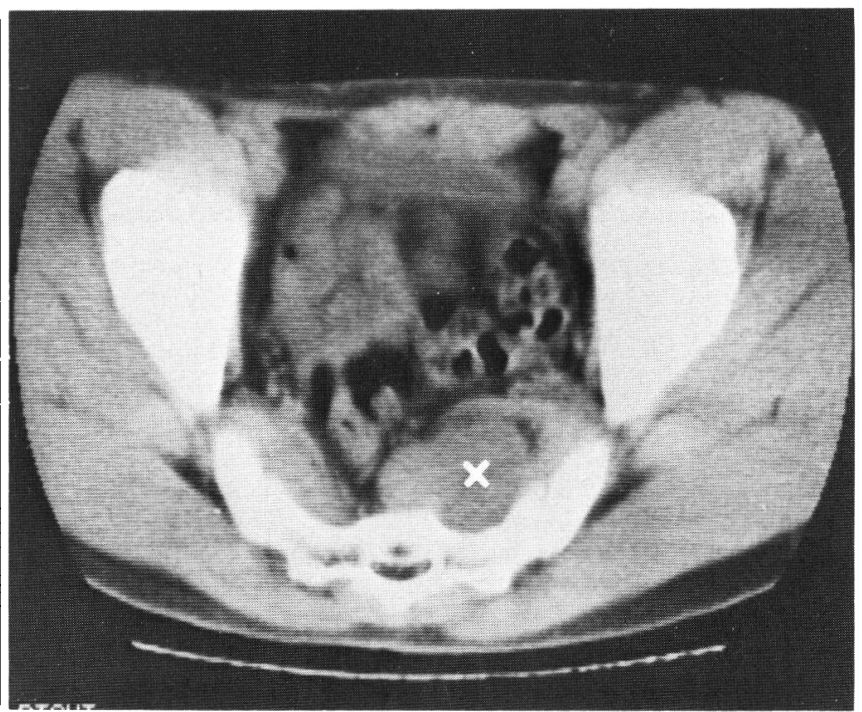

Figure 6-Patient 9. CT scan of pelvis: A pre-sacral soft tissue mass (hemangiopericytoma) is indicated by ' $x$ '.

\section{Discussion}

We have found CT scanning to be of value in investigating mass lesions affecting three particular areas of the peripheral nervous system: the lumbar and sacral nerve roots and plexuses, the brachial plexus, and proximal individual nerve trunks.

In the investigations of these patients, CT scanning was compared to conventional radiologic procedures and isotope bone scanning and it was found that: 1 . In four patients (with five lesions) CT scanning was the only procedure to demonstrate the tumor. Not surprisingly, these were the patients in whom the lesion had not arisen from or did not involve bony structures, and hence the plain radiographs and isotope bone scans were normal. 2. Another group (two patients) were those with abnormalities of uncertain significance on plain radiographs, and normal bone scans, but in whom CT scanning unequivocally showed a lesion. 3 . The final group of three patients were those with lesions arising from or involving bony structures and therefore the plain radiographs and bone scans were clearly abnormal. However the true anatomical extent of not only the bony involvement but the soft tissue mass was demonstrated only by CT scanning. 


\section{Lumbar and sacral root and plexus lesions}

The role of CT scanning in radiculopathies due to herniated intervertebral discs is well established (Meyer et al., 1979; Carrera et al., 1980; Hochman et al., 1980). The usefulness of CT scanning in investigating patients with radiculopathy caused by infiltrative spinal malignancy has received less attention although Federle et al. (1980) have reported 10 patients with "sciatica" which was unexplained by routine investigations. Seven had normal or equivocal radiographs of the lumbo-sacral spine and five had normal or equivocal myelograms. CT scanning showed destructive lesions primarily of the sacrum in every case ( 9 patients had malignant tumors, 1 had tuberculous osteomyelitis). Our patients 1, 2, and 3 (Figures 1,2) were similar to those reported by Federle et al. in that they had destructive lesions of the sacrum producing nerve root infiltration and compression. More difficult to demonstrate however, are those malignancies not arising from bone that involve the lumbar roots or plexus in the retroperitoneal area (e.g. patient 4a). We agree with Stephens et al. (1977), Weis et al. (1978), and Donovan et al. (1981) that CT scanning is now the investigation of choice for mass lesions in this region. Retroperitoneal hematomas are also well demonstrated by CT scanning (Emery and Ochoa, 1978; Massey, 1981).

\section{Brachial plexus}

Two of our patients ( 5 and 6 ) had typical Pancoast syndromes (superior pulmonary sulcus tumors causing a Horner's syndrome and symptoms due to invasion of the lower brachial plexus), and patient $4 \mathrm{~b}$ had neoplastic infiltration of the lower trunk of the plexus without a Horner's syndrome. Plain radiography even with special apical views were normal, in accord with other reports of patients with Pancoast tumor (Simon et al., 1964; Hepper, 1966; Paulson, 1975). Our experience therefore shows that in these patients with normal plain radiographs and Pancoast tumors, CT is the imaging technique of choice. Cascino et al. (1982) have also pointed out the value of CT scanning in the diagnosis of metastatic tumors causing brachial plexopathy. Negative $\mathrm{CT}$ scans of the brachial plexus region can also provide useful diagnostic and prognostic information. We have one patient, a heavy smoker, who presented with a lesion of the lower trunk of the brachial plexus (but without Horner's syndrome). Full investigations including CT scanning failed to show any infiltrative or compressive cause. He was presumed to have idiopathic brachial plexus neuropathy in an atypical distribution, and this diagnosis was borne out by his subsequent spontaneous improvement.

\section{Proximal peripheral nerves}

Three of our patients $(7,8,9)$ had proximal lesions of various peripheral nerves (sciatic and inferior gluteal, femoral and lateral femoral cutaneous nerves, and posterior cutaneous nerve of the thigh). In proximal mononeuropathies, like lumbo-sacral radiculopathies and plexopathies, the underlying cause may be difficult to detect by plain radiography and isotope bone scanning if the mass causing the compression does not arise from or involve bone (eg. patient 7; Figure 4). Previous reports of CT scanning demonstrating the etiology of proximal compressive mononeuropathies include intrapelvic hematomas causing femoral (Simeone et al., 1977; Heilbronn et al., 1979; Zarranz and Salisachs, 1979; Lazaro et al., 1981) and sciatic (Wallach and Oren, 1979) neuropathies, and of a malignant schwannoma of the sciatic nerve (Weis et al., 1978). CT scanning has also been used to demonstrate a lipoma causing a radial neuropathy (Millefiorni et al., 1980) and neurofibromas in the extremities (Laursen and Reiter, 1980).

In conclusion, CT scanning may be invaluable in the investigation of patients with mass lesions compressing or damaging nerve plexuses and proximal peripheral nerves. Its particular value is in demonstrating soft tissue masses that do not arise from or involve bone, and therefore are not seen on plain radiographs or isotope bone scans.

\section{ACKNOWLEDGEMENTS}

We thank Dr. Calvin Melmed for helpful discussion and Arlene Schinasi for the preparation of the manuscript.

\section{REFERENCES}

Carrera, G.F., Williams, A.L., Haughton, V.M. (1980). Computed tomography in sciatica. Radiology, 137:433-437.

Cascino, T.L., Kori, S.H., Krol, G., Foley, K.M. (1982). Brachial plexopathy in cancer patients: Diagnostic usefulness of CT scans. Neurology (NY), 32:A 74 (abst. P. 45).

Donovan, P.J., Zerhouni, E.A., Siegelman, S.S. (1981). CT of the psoas compartment of the retroperitoneum. Semin. Roentgenol., 16:241-250.

Emery, S., Ochoa, J. (1978). Lumbar plexus neuropathy resulting from retroperitoneal hemorrhage. Muscle \& Nerve, 1:330-334.

Federle, M.P., Moss, A.A., Margolin, F.R. (1980). Role of computed tomography in patients with "sciatica". J. Comput. Assist. Tomogr., 4:335-341.

Heilbronn, Y.D., Williams, V.L., Kranzler, L.I., Banuchi, F.F., Sigueira, E.B. (1979). CT scan of retroperitoneal hematoma with neuropathy. Surg. Neurol., 16:251-252.

Hepper, N.G., Herskovic, T., Witten, D.M., Mulder, D.W.. Woolner, L.B. (1966). Thoracic inlet tumors. Ann. Int. Med., 64:979-989.

Hochman, M.S., Pena, C., Ramirez, R. (1980). Calcified herniated thoracic disc diagnosed by computerized tomography. J.Neurosurg., 52:722-723.

Laursen, K., Reiter, S. (1980). Computed tomography in soft-tissue disorders of the lower extremities. Acta. Orthop. Scan., 51:881-885.

Lazaro, R., Rinker, R., Weiss, J., Oleijnizak, S. (1981). Femoral and obturator neuropathy secondary to retroperitoneal hemorrhage: The value of the CT scan. Comput. Tomogr., 5:221-224.

Massey, W.E. (1981). CT evaluation of lumbosacral plexus disorders. Postgrad. Med., 69:116-118.

Meyer, G.A., Haughton, V.M., Williams, A.L. (1979). Diagnosis of herniated lumbar disc with computed tomography. N. Eng. J. Med., 301:1166-1167.

Millefiorini, M., et al. (1980). A case of radial nerve paralysis caused by lipoma: Role of CT in diagnosis and clinical aspects. Rev. Neurol., 50:406-409.

Paulson, D.L. (1975). Carcinomas in the superior pulmonary sulcus. J. Thorac. Cardiovasc. Surg., 70:1095-1102.

Simeone, J.F., Robinson, F., Rothman, S.L., Jaffe, C.C. (1977). Computerized tomographic demonstration of a retroperitoneal hematoma causing femoral neuropathy. J. Neurosurg., 47:945-948.

Simon, H., Moon, A.D. (1964). Pitfalls in the diagnosis of Pancoast tumors. Radiology, 82:235-239.

Stephens, D.H., Sheedy, P.F., Hattery, R.R., Williamson, B. (1977). Diagnosis and evaluation of retroperitoneal tumors by computed tomography. Am. J. Roentgenol., 129:395-402.

Stewart, J.D., Murphy, G.R., Wee, R. (1981). Sciatic nerve palsy due to endometriosis. Can. J. Neurol. Sci, 8:202.

Wallach, H.W., Oren, M.E. (1979). Sciatic nerve compression during anticoagulation therapy: Computerized tomography aids in diagnosis. Arch. Neurol., 36:448.

Weis, L., Heelan, R.T., Watson, R.C. (1978). Computed tomography of orthopedic tumors of the pelvis and lower extremities. Clin Orthop., 130:254-259.

Zarranz, J.J., Salisachs, P. (1979). Femoral neuropathy due to compression by retroperitoneal hemorrhage: A modern evaluation. J. Neurol. Sci., 43:479-482. 